\title{
CONSTRUCTION PLANEX: A Knowledge Intensive Planner for Construction Projects
}

\author{
Carlos Zozaya-Gorostiza, Chris Hendrickson, Daniel R. Rehak and Peter Lim \\ Department of Civil Engineering \\ Carnegie Mellon University \\ Pittsburgh, PA 15213-3890 \\ USA
}

\begin{abstract}
This paper reviews the capabilities and application of the CONSTRUCTION PLANEX system. This knowledge-based expert system assists in the selection of construction technology, the definition of work tasks, the estimation of required resources and durations, the estimation of costs, and the preparation of a project schedule. The system has been applied in laboratory simulations in three ways: interactively as an intelligent assistant, independently as an automated planner, and as part of an integrated building design environment. CONSTRUCTION PLANEX can plan for the excavation and structural erection of concrete and steel buildings; example applications have ranged from two to twenty stories. Provisions are included for expanding the knowledge base to consider other functional elements or facility types. Descriptions of the system architecture, knowledge representation scheme, control procedures, and applications are included.
\end{abstract}

\section{Introduction}

The construction planning process involves the definition of work tasks, the choice of construction technologies, the estimation of durations and costs for individual tasks, and the preparation of project schedules. It may be regarded as an example paradigm of the more general project planning process in which the product to be built is not necessarily a constructed facility.

The first motivation for studying the construction planning process is that it is both a crucial and a challenging task in the management of construction projects. It is crucial to the eventual success of a project because project control and monitoring is based on a particular project plan. Poor estimates or schedules can easily result in large construction cost increases or delays. Similar effects may be obtained because of inappropriate or inconsistent decisions concerning the technologies to be used when performing the work tasks. Also, it is a challenging process because the planner is concerned not only with the generation of a feasible plan, but with the formulation of a good one. There may be numerous constraints that complicate the planning process such as those related with the availability of resources, the completion times for the work tasks or the limitations on project budget.

The second motivation is that despite its importance and complexity, little attention has been paid to analyzing the process by which project plans are or should be formed. Current construction planning relies upon manual formulation of plans and is usually performed in an intuitive and unstructured fashion with considerable reliance on engineering judgment. Few project planning aids exist other than general project templates or past project networks that can be adapted to the particulars of a new project. During the last few years, several expert systems in the area of construction engineering and management have been developed, or are under development ${ }^{1}$. Most of these systems are experimental prototypes that have not yet been used in practice. Existing computer tools are applicable to only some of the parts of the whole process. For example, commercial scheduling systems require a complete specification of the project network as an input. When using these systems, decisions concerning plan formulation and refinement are made separately from project scheduling decisions. Developing an integrated computer tool for the complete planning process provides a unified framework for analyzing the interdependencies of planning decisions.

Finally, another motivation for studying the construction planning process is that it presents many similarities with other types of planning processes. For example, there are many parallels between the tasks followed by a construction planner and those followed by process planners in manufacturing. Process planners have to identify and sequence machine operations for manufacturing specific products. Construction planners have to identify and sequence construction activities for building specific facilities. Therefore, some of the results from the development of CONSTRUCTION PLANEX are applicable to the more general problem of project planning.

CONSTRUCTION PLANEX is a knowledge-based expert system that assists in the generation of project plans for concrete and steel buildings. In developing this system, tools for representing project planning knowledge, applying this knowledge to perform planning decisions and controlling the execution of the planning process were developed. These tools were incorporated into a generic knowledge-based system architecture for project planning, called PLANEX, which has been used in various application domains.

The development of the PLANEX architecture and the CONSTRUCTION PLANEX system alternated between conceptualization and implementation. At the beginning of the study, it was difficult to design a generic architecture for project planning without having explored the application of expert systems to the construction planning process. Our first major task was to develop a prototype expert system for excavation tasks. Experiences learned from developing this prototype were used to abstract the problem and to formulate general architectural concepts for PLANEX. These concepts were implemented in a first version of the CON- 
STRUCTION PLANEX system ${ }^{2}$ which was capable of planning the excavation and erection of modular concrete buildings. Additional modifications to this second prototype led to the architecture of PLANEX and to the current version of the CONSTRUCTION PLANEX system. ${ }^{3}$ CONSTRUCTION PLANEX is implemented using the Knowledgecraft ${ }^{\mathrm{TM}}$ expert system development environment.

\section{Models for Construction Planning Used in the System}

\subsection{Definition of Work Tasks and Precedence Relationships}

In generating a project activity network, CONSTRUCTION PLANEX uses a "bottom-up" approach that includes five steps:

1. Describe Building. The building is described in terms of unitary components called design elements.

2. Identify Activities. The system determines which activities are required to construct each design element. These tasks are called the element activities of the design element.

3. Aggregate Activities. The system aggregates element activities into more manageable tasks called project activities.

4. Estimate Attributes. The system estimates durations and costs associated with particular project and element activities.

5. Link Activities. The system establishes precedence relationships among project activities in order to create a project activity network.

The first three steps of the process are inspired by Baracco's model for integrating duration and costs. This model differs from the "top-down" approach used in other activity formulation models in which an aggregate activity network is expanded successively. The reasons for using the model to identify project activities are the following:

- the "bottom-up" approach has many similarities with the cost estimating process routinely used by contractors;

- construction knowledge is more easily available in the "bottom-up" approach because cost estimating books and construction technology literature usually focus on building components; and

- a "top-down" approach would eventually reach a detail level corresponding to the element activity level of CONSTRUCTION PLANEX in order to perform quantity-take-off calculations.

\subsection{Choice of Technologies and Construction Methods}

CONSTRUCTION PLANEX assigns crews to activities after formulating the project activity network and before estimating activity durations and costs. Each crew is a package composed of labor and equipment. CONSTRUCTION PLANEX models the human technology selection process in two steps:

1. Select Crew Type. The system chooses a crew schema for each project activity by using information about site characteristics, design elements and construction activities. In this step, CONSTRUCTION PLANEX assumes that each activity can be performed with its most appropriate crew.

2. Decide the Number Crews to be Used. Once the crew type has been determined, the system decides how many crews will be used to perform the activities by using knowledge about their recommended durations. These durations are a function of the total amount of work to be performed on each activity. In this step, no time-cost trade-offs are analyzed.

\subsection{Estimation of Activity Durations and Costs}

CONSTRUCTION PLANEX estimates durations and costs of project activities using simple models based on average productivities and unit costs ${ }^{5}$. Activity durations are computed following the process indicated in Figure 1. First, the system computes the expected duration of the activity in hours. After the total hours have been computed, the system computes the duration of the activity in days. Following this computation, CONSTRUCTION PLANEX eliminates day fractions whenever overtime hours are permitted. This is done for the purpose of avoiding payment of a full extra day of salary to the allocated crew. Finally, the system computes the distribution of normal and overtime hours. Expected activity costs are estimated using the duration estimates and unit costs.

A desirable extension to the basic CONSTRUCTION PLANEX system would be to replace the simple non-hierarchical estimation procedures described above with a more elaborated hierarchical estimator that uses the concepts developed in MASON6. Also, it might be desirable to include procedures for computing not only the expected values of these quantities, but also some measure of their variability. This would allow the system to use probabilistic scheduling models such as the PERT for estimating expected project durations.

\subsection{Preparation and Maintenance of Project Schedules}

In preparing and maintaining project schedules, CONSTRUCTION PLANEX uses an activity network model that differs from the conventional Activity-On-Node (AON) and Activity-On-Branch (AOB) models ${ }^{7}$. In this model, each activity is represented by a start node, a finish node and an intervening link which can represent an activity, a precedence relationship or a window time constraint. This activity network model, which we call the unified model, permits up to sixteen precedence and window types and allows use of standard longest path algorithms (e.g., the basic Critical Path Method) in solving for activity and event earliest and latest times. The model can also be used to model activity splitting. Activity splitting allows work to commence, stop and then re-commence on an activity. The development of the unified network model was motivated by the desire of having an activity network model in which time constraints could be solved using simple scheduling procedures. The model accomplishes this objective and provides CONSTRUCTION PLANEX with a means for computing activity floats when various types of precedence relationships are present. 


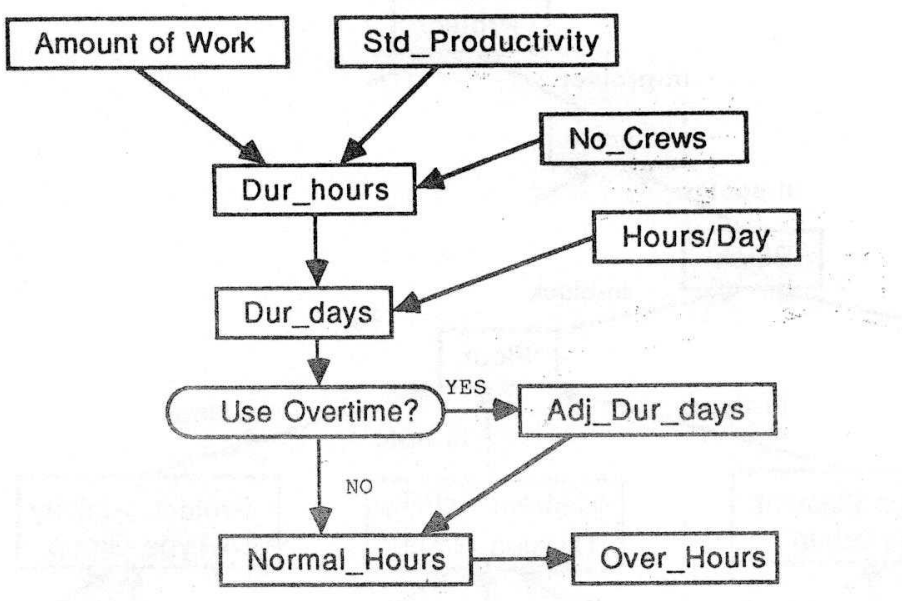

Figure 1: Process for Activity Duration Estimation

\section{Overview of the CONSTRUCTION PLANEX Architecture}

\subsection{Representation Structures}

Information about solution elements, planning decisions and input data for the planning process is stored in a global data store called the context. The context consists of different hierarchical representation structures that are composed of objects linked with each other using different types of relations. In this article, the terms object, frame and schema are used interchangeably. Each object has a unique name and contains slots that store values about particular attributes of the object or pointers to other context objects. An object may belong to more than one hierarchical structure. Also, there are links for relating different context structures.

CONSTRUCTION PLANEX has the three basic representation structures shown in Figure 2:

- Tree of Design Elements. The building is described in terms of individual design element schemas that represent building components such as beams or columns. These schemas are aggregated with respect to their material (concrete or steel), type (e.g., column) and location (e.g., first floor).

- Tree of Element Activities. The activities required to construct each of the design elements are aggregated by using the MASTERFORMAT coding system ${ }^{8}$ into a tree of element activities.

- Tree of Project Activities. Construction activities are aggregated with respect to the type of activity they represent (e.g. formwork) and the type of design element associated with them (e.g. formwork in columns versus formwork in beams).

\subsection{Operators}

Solution to the project planning problem is achieved by applying problem-solving operators. Each operator is a procedural function that modifies the context by creating, modifying or deleting objects. There are two types of operators: domain and control operators. Domain operators perform specific planning tasks such as technology choice, activity duration estimation, or scheduling. Control operators decide the sequence in which domain operators are executed. Control operators are used to implement different control strategies for solving the problem.

CONSTRUCTION PLANEX has three basic types of problem-solving operators:

- design element operators;

- element activity operators; and

- project activity operators.

This classification is based on the type of context objects that the operators take as arguments. For example, the operator get-duration-pas acts on project activity schemas. Therefore, it is considered to be a project activity operator.

For example, CONSTRUCTION PLANEX has eight operators that act on project activity schemas:

- create-pa-tree creates the tree of project activities; 


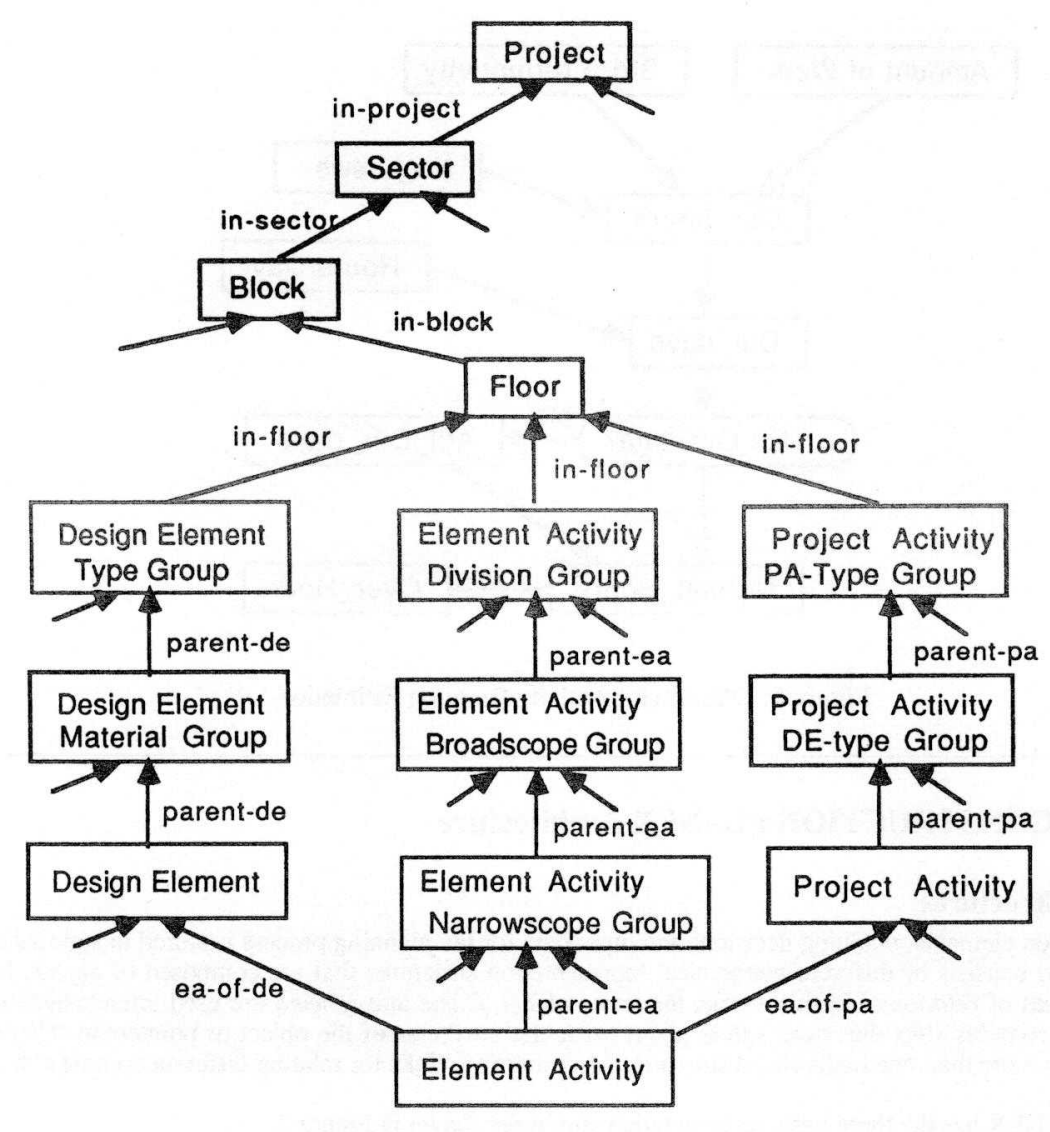

Figure 2: Representation Structures of CONSTRUCTION PLANEX

- select-technology-pas chooses crew types for activities;

- compute-amount-pas computes the quantities-take-off for the project activities;

- determine-recommended-duration-pas recommends an appropriate duration in days for the activities;

- get-duration-pas decides how many crews are allocated to the activities and obtains the number of normal and overtime working hours;

- get-successors-pas establishes precedences among project activities, based upon the geometry, location and type of the underlying element activities;

- get-lags-pas computes the leads and lags among successive project activities;

- get-cost-pas estimates the cost of the activities.

In addition, the system has the following two operators that operate on the complete set of project activities:

- the floyd-warshall algorithm ${ }^{9}$, that finds the earliest and latest start and finish times for the project activities; and

- the compute-npv operator, which obtains the net present value of a project using scheduling and financial information.

\subsection{Knowledge Sources}

Knowledge required by the operators is stored in groups of rules called Knowledge Sources (KS's). Each KS provides information for particular operations such as activity formulation, duration estimation or precedence determination. Knowledge sources may be used not only to return values to the operators, but also to modify the context and to invoke the evaluation of other KS's. The Knowledge Base of the system is composed of many KS's.

The Knowledge Base of CONSTRUCTION PLANEX is composed of the following types of knowledge sources: 
- Element Activity KSs indicate the set of activities required for constructing a design element;

- Amount KSs indicate the formulas to be used when computing the amount of work for each element activity;

- Unit $K S s$ indicate the default unit of measure to be used for each type of element activity;

- Material KSs specify the set of materials required for performing an element activity;

- Project Activity $K S$ s indicate the name of the project activity appropriate for grouping a particular element activity;

- Technology KSs recommend appropriate crews for performing project activities;

- Duration KSs indicate a desirable duration for project activities;

- Successor KSs indicate the name of the successors of particular project activities; and

- Lag KSs indicate the type and values of lead and lags between two succeeding project activities.

An example of an element activity KS is shown in Figure 3 as a decision table used in the original acquisition or representation phase. The actual schema used by this KS appears in Figure 4. This KS returns the names of the element activities required to build a column footing and their codes. The type of this KS is "all", meaning that all the rules should be fired sequentially. The cond-objects slot indicates that all the information required to evaluate the $\mathrm{KS}$ is stored in the design element schema being analyzed (the "current-object") and the "soil-info" object. The KS of Figure 4 contains four conditions:

- the first condition binds the value of the root-code slot of the design element object to the variable "<roob";

- the second condition checks whether the type-de slot of the design element has a value equal to 60 ;

- the third condition binds the value of the number-de slot of the design element to the variable "<number "; and

- the fourth condition checks whether the possible-use slot of the soil-info object has a value equal to "backfill".

Also, the KS has three rules:

- the first rule indicates that all concrete column footings require excavation, formwork, reinforcement, pouring and stripping;

- the second rule indicates that if the soil is appropriate for backfilling, constructing the footing requires piling up the excavated material in order to use it again;

- Finally, the third rule indicates that if the soil is not appropriate for backfilling, the contractor has to dispose of the excavated material and borrow material from a different source.

\begin{tabular}{|c|c|c|c|}
\hline \multicolumn{4}{|l|}{$\begin{array}{l}\text { Element Activity KS for } \\
\text { Concrete Column Footings }\end{array}$} \\
\hline CONDITIONS & \multicolumn{3}{|c|}{ RULES } \\
\hline current-object is a cast-in-place concrete column footing & $\mathrm{T}$ & $T$ & $T$ \\
\hline soil is appropriate for backfilling & 1 & $T$ & $\mathrm{~F}$ \\
\hline excavate-column-footing & $x$ & 1 & I \\
\hline dispose-excavation-column-footing & 1 & 1 & $\mathrm{x}$ \\
\hline pile-up-excavation-column-footing & 1 & $\mathrm{x}$ & 1 \\
\hline borrow-material-column-footing & 1 & 1 & $\mathrm{x}$ \\
\hline place-forms-column-footing & $\bar{x}$ & $T$ & 1 \\
\hline reinforce-column-footing & $\mathrm{x}$ & $\mathrm{T}$ & 1 \\
\hline pour-concrete-column-footing & $\mathrm{x}$ & 1 & 1 \\
\hline remove-forms-column-footing & $\bar{x}$ & $T$ & 1 \\
\hline
\end{tabular}

Figure 3: Example of a KS for Element Activity Creation

\subsection{User Interface}

CONSTRUCTION PLANEX is an interactive system architecture that provides the user with an interface for modifying any of the three components described above, for changing the problem-solving behavior of the system or for inserting planning decisions. The user interface is composed of several editors, command menus, graphic displays and a report generator. As an example, the following user interface mechanisms will be discussed: the GANTT Interactive Scheduler, output graphics, output reports and explanation functions. 


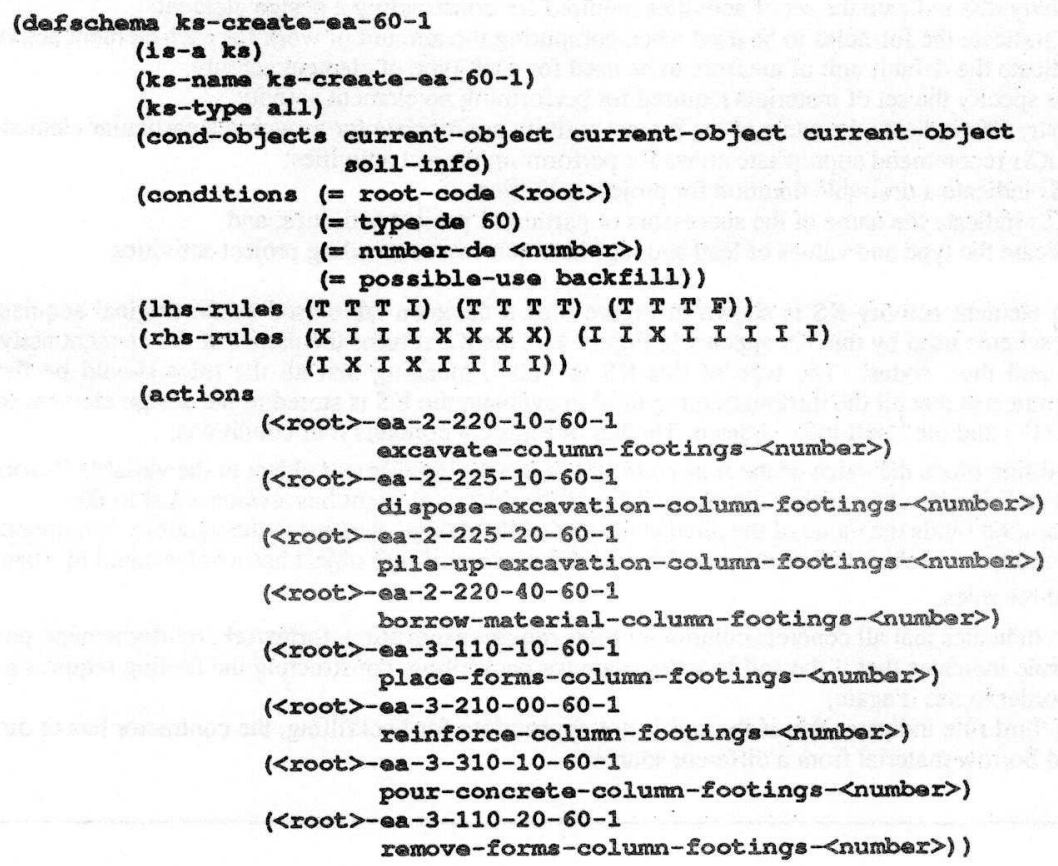

Figure 4: Example of a KS Schema Code for Element Activity Creation

Interactive graphics provide the user with a useful mechanism for modifying the information stored in domain objects. An example of this type of user interaction mechanism is the GANTT Interactive Scheduler (GANTT). GANTT allows the user to calculate, display and modify scheduling information for a particular set of activities. Figure 5 shows a screen dump of GANTT. Schedule changes are performed by clicking the mouse on the bars representing the activities. Depending on the type of click used, an activity may be shortened, expanded or shifted to a different place. In addition, the user may modify activity precedences, time scales and resource trigger levels by choosing menu options.

Menus provide the user with means for invoking the execution of domain and control operators. In CONSTRUCTION PLANEX, menus are used for various purposes:

- to indicate the name of an operator to be executed;

- to specify in which context objects this operator should perform;

- to set values of global variables; or

- to answer a question that has a predefined set of answers.

In addition to the graphics used in GANTT, CONSTRUCTION PLANEX produces the following output graphics:

- Activity-On-Node diagram, which displays the project activity network with some scheduling information such as activity name and start times;

- Cost Curve, which displays the total activity cash flows versus time on the project;

- Cumulative Cost Curve, which displays cumulative cash flows of project activities; and

- an animation program, called ANIMATOR, which displays the status of the building at various points of the project execution.

The first three output graphics are an integrated portion of CONSTRUCTION PLANEX. ANIMATOR is a stand-alone threedimensional graphics program running on an IRIS-2400 graphics computer. ANIMATOR simulates the manner in which a building is being constructed. The input to this program is a file created by CONSTRUCTION PLANEX system which contains information about the starting and ending times of each building component. ANIMATOR displays building components incrementally using various colors for representing different construction activities. During the simulation, a clock shows the number of days that have elapsed since the beginning of the project.

CONSTRUCTION PLANEX has a variety of reports that may be used to obtain information about context objects. As an example, consider the output reports shown in Figures 6 to 8: 


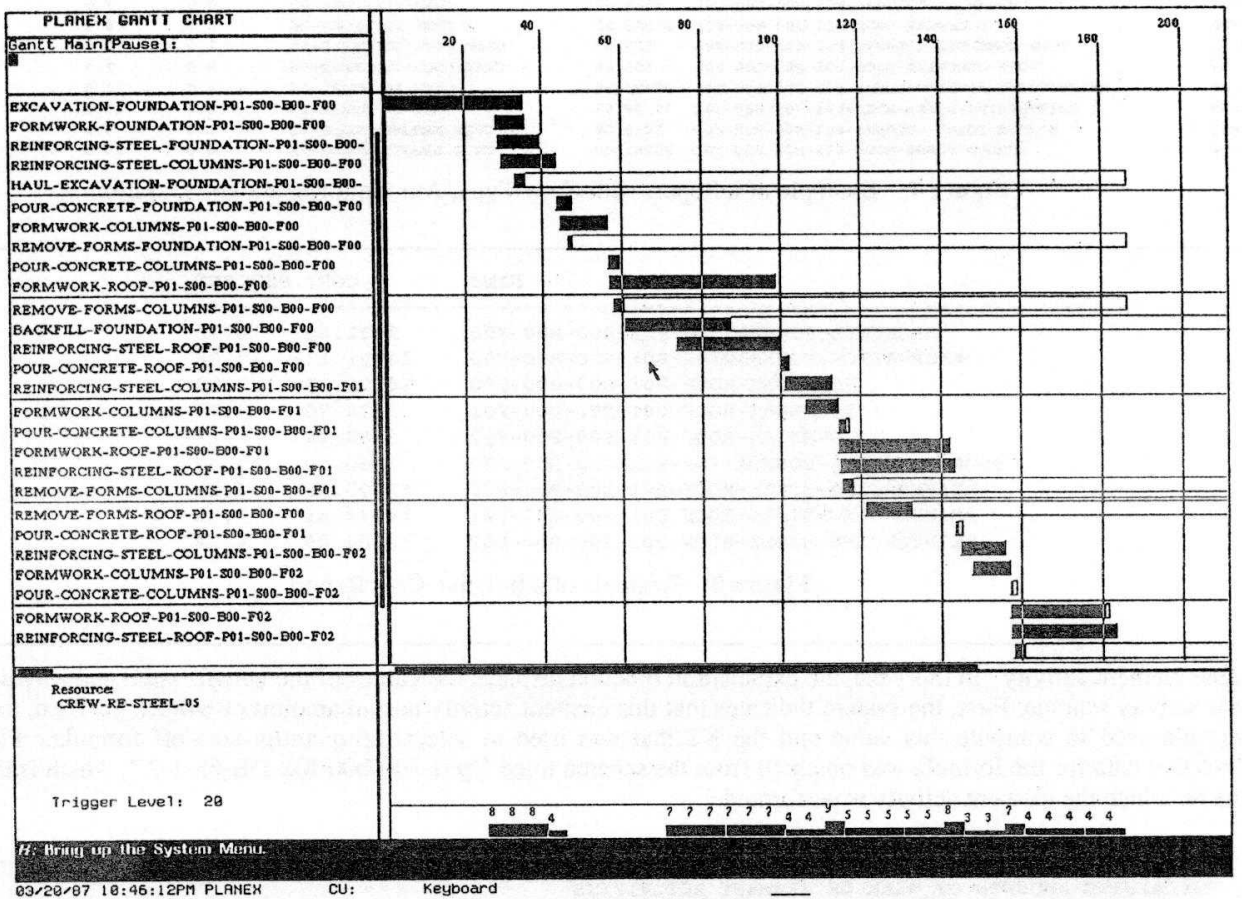

Figure 5: Screen of the GANTT Interactive Scheduler

- The first report shows the values of the scheduling slots of those project activities whose earliest start time (EST) are less than 60 days. In this report activities are sorted with respect to their EST.

- The second report shows the crew types of the project activities located in the first floor of the building (floor "f01"). In this report activities are sorted with respect to their names. The report shows that the formwork activities of the columns are done with a different crew type ("crew-formwork-05") than the corresponding activities in roof elements ("crew-formwork-06"). A similar situation occurs with the activities for pouring concrete in a particular floor.

- Finally, the third report shows how much each project activity contributes to the total project cost. In this report activities are also sorted by name, and only those activities with a percentage cost greater than $4 \%$ are printed.

\begin{tabular}{|c|c|c|c|c|c|c|}
\hline CODE & NAME & DURATION & EsT & EFT & LST & LFT \\
\hline $10-60$ & EXCAVATION-FOUNDATION-P01-SOO-BOO-FOO & 35.0 & 0.00 & 35.00 & 0.00 & 35.00 \\
\hline $20-60$ & FORMWORK-FOUNDATION-PO1-SOO-BOO-FOO & 7.0 & 27.94 & 35.54 & 27.94 & 35.54 \\
\hline $40-60$ & RUEINFORCING-STEEL-FOUNDATION-P01-SOO-BOO-F00 & 11.0 & 28.48 & 39.48 & 28.48 & 39.85 \\
\hline $80-65$ & REINFORCING-STEEL-COLUMNS-P01-SO0-BOO-FOO & 14.0 & 29.49 & 43.49 & 29.49 & 43.49 \\
\hline $15-60$ & HAUL-EXCAVATION-FOUNDATION-P01-SOO-BOO-FOO & 2.0 & 32.70 & 35.61 & 184.26 & 186.26 \\
\hline $50-60$ & POUR-CONCRETE-FOUNDATION-P01-SOO-BOO-FOO & 4.0 & 43.49 & 47.49 & 43.49 & 47.49 \\
\hline $20-65$ & FORMWORK-COLUMNS-POI-SOO-BOO-FOO & 12.0 & 44.49 & 56.49 & 44.49 & 56.49 \\
\hline $30-60$ & REMOVE-FORMS-FOUNDATION-P01-S00-BOO-FOO & 1.0 & 46.13 & 47.56 & 185.26 & 186.26 \\
\hline $50-65$ & POUR-CONCRETE-COLUMNS-POI-SOO-BOO-FOO & 3.0 & 56.49 & 59.49 & 56.49 & 59.60 \\
\hline $20-80$ & FORMWORK-ROOF-P01-S00-B00-F00 & 42.0 & 56.55 & 98.55 & 56.55 & 98.55 \\
\hline $30-65$ & REMOVE-FORMS-COLUMNS-P01-S00-B00-F00 & 3.0 & 57.89 & 60.49 & 183.26 & 186.26 \\
\hline
\end{tabular}

Figure 6: Example of a Scheduling Report

CONSTRUCTION PLANEX provides the user with some explanation about the causes of particular results. The current version of the system has no general explanation mechanism but does provide a set of specific explanation functions for each of the attributes of a context object. Figure 9 shows the information displayed when the user asks for an explanation about the amount of work of a 


\begin{tabular}{|c|c|c|c|c|c|}
\hline CODE & IRAM & ASOONRT & Crum & 200. & DURArrom \\
\hline $20-65$ & 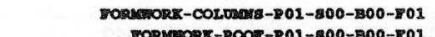 & 5040.00 & 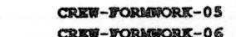 & 6.0 & 8.0 \\
\hline $\begin{array}{l}20-80 \\
50-65\end{array}$ & 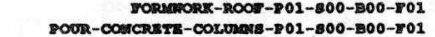 & $\begin{array}{r}20261.00 \\
130.67\end{array}$ & 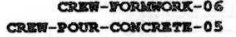 & $\begin{array}{l}6.0 \\
1.0\end{array}$ & $\begin{array}{r}28.0 \\
2.0\end{array}$ \\
\hline 50-80 & 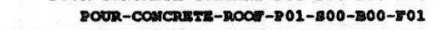 & 504.16 & 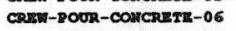 & 3.0 & 1.0 \\
\hline $40-65$ & 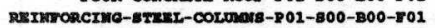 & 27227.36 & CREWT-RE-ATYARL-05 & 4. 0 & 12.0 \\
\hline $40-80$ & PRTMRORCIWC-8IREL-ROOP-P01-800-800-701 & 79328.57 & CEST-RE-8TERL-05 & 5.0 & 28.0 \\
\hline $\begin{array}{l}30-65 \\
30-80\end{array}$ & 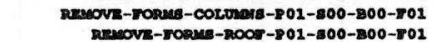 & $\begin{array}{r}5040.00 \\
20261.00\end{array}$ & 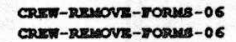 & $\begin{array}{l}4.0 \\
4.0\end{array}$ & $\begin{array}{r}3.0 \\
12.0\end{array}$ \\
\hline
\end{tabular}

Figure 7: Example of a Report with Crew Type, Amount of Work and Duration

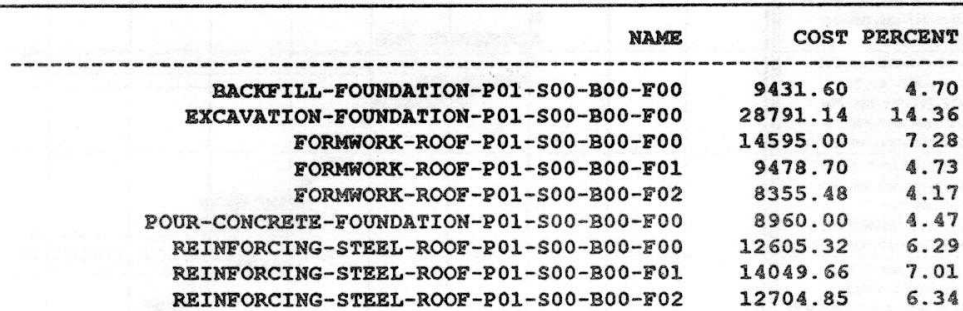

Figure 8: Example of a Relative Cost Report

particular element activity. In this case, the explanation function displays the values of the why-formula and why-amount slots of the element activity schema. First, the system indicates that this element activity has an amount of $648.27 \mathrm{lb}$. Then, the system displays the formula used to compute this value and the KS that was used to select the quantity-take-off formula. Finally, the system indicates that data for the formula was obtained from the schema titled "p01-s00-b00-f02-DE-65-1-2", which is the design element schema on which the element activity is performed.

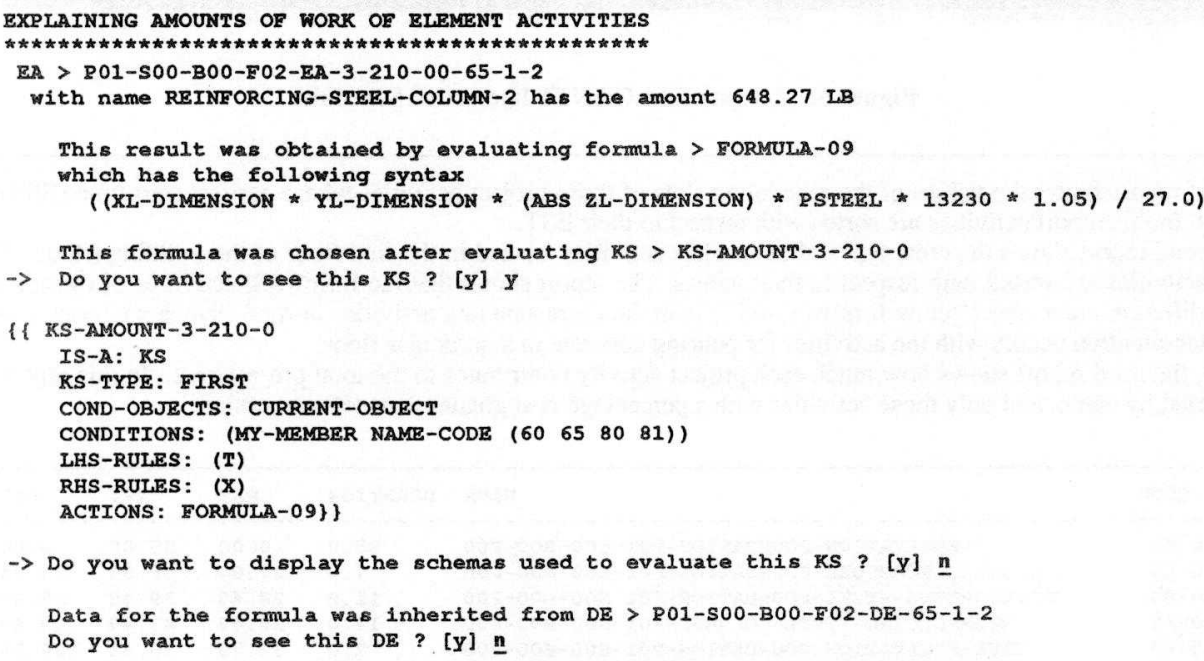

Figure 9: Example of Explanation for Quantities-Take-Off

\subsection{Relationships Among Architectural Components}

Figure 10 illustrates the relationships between an operator, its associated knowledge sources and the different types of context objects related to the operator. Each operator may be applied to different sets of objects called the application objects of the operator. The performance of an operator may be sequential or simultaneous to all of the application objects. During a sequential application, the object where the operation is being performed is called the current object. When an operator is executed, a set of knowledge sources may be evaluated and the results of this evaluation are returned to the operator. For each operator, the context may be divided into three different sets of objects: the input objects, the output objects and the remaining objects that are not related with the operator. The input objects of the operator contain all the data required to evaluate the knowledge sources related with the 
operator and to perform procedural calculations. The objects affected by the application of an operator and the evaluation of its knowledge sources constitute the output objects of the operator. The sets of input and output objects may have some common elements. Usually, the current object belongs both to the input and output objects.

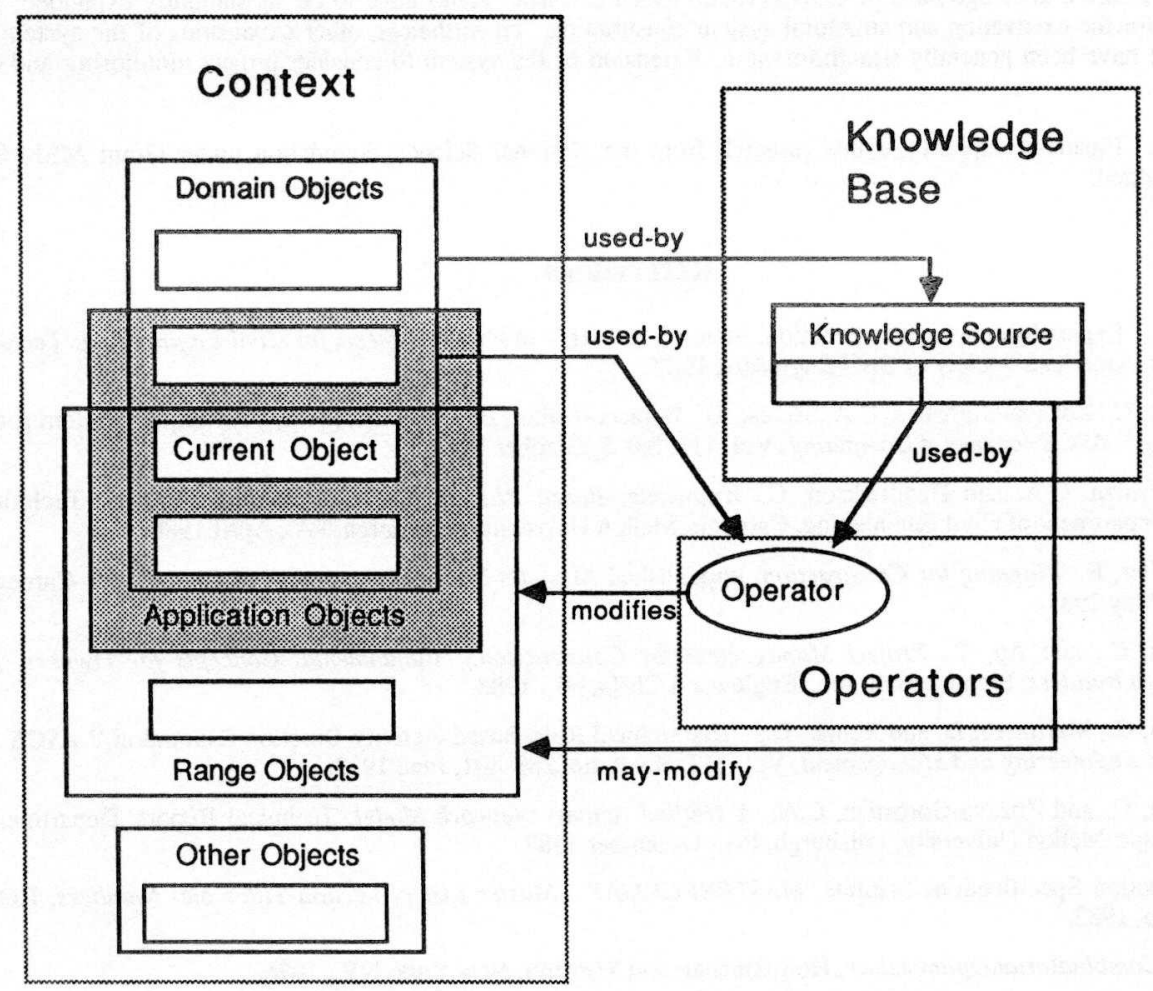

Figure 10: Relationships of Operators, Knowledge Sources and Context Objects

The set of context changes caused by a domain operator are known as the effects of the operator. Effects are described as changes to slot values of particular objects. An effect is predictable if it can be described before the operator is executed, and it is unpredictable otherwise. The information about predictable and unpredictable effects of domain operators is stored in other context objects that contain declarative knowledge about the operators themselves. These objects are called the Domain Operator Schemas (DOSs) associated with the operators. The information stored in the DOSs is used by the control operators to decide which planning tasks should be performed at different points of the planning process. Some control operators plan sequences of domain operators, while others are responsible for executing the (operator object) pairs and recording unpredictable effects. The user, by means of a menu-driven interface, may also modify the information of the agenda (e.g. by inserting goals to be achieved or changes to be propagated) or invoke the execution of domain or control operators.

\section{Use of CONSTRUCTION PLANEX}

CONSTRUCTION PLANEX has been used in three different ways:

- interactively as an intelligent assistant;

- as an automated planner; and

- as a component of the Integrated Building Design Environment (IBDE) ${ }^{10}$.

In these applications, CONSTRUCTION PLANEX has been used for planning the excavation and erection of about fifteen different buildings. Most of these buildings are concrete office buildings. In one of the examples tested, an actual building was coded and entered into the system. Information for this example was obtained from a local contractor. In other runs, buildings were input using the Input Generator program. Finally, a third set of examples were obtained from other knowledge-based systems of the IBDE. The 
total time spent by the system in generating an initial project plan ranged from 10 minutes to 90 minutes, depending upon the complexity of the building. In these runs, scheduling was the most time consuming operation.

The development of CONSTRUCTION PLANEX has shown that an integrated system for construction planning is feasible. Important characteristics of the system include the bottom-up planning approach based on individual design elements, the grouping of rules in knowledge sources, and the hierarchical organization of planning information. An expert system aid for planning of this type could yield substantial benefits in producing more consistent and detailed plans in a less costly fashion than traditional methods.

For field application, the knowledge base in CONSTRUCTION PLANEX would have to be substantially expanded; the current version only plans for the excavation and structural system construction. Nevertheless, other expansions of the system to include steel frame erection have been generally straightforward. Extension of the system to consider project monitoring and control are also possible.

Acknowledgments. Financial support for this research from the National Science Foundation under Grant MSM-8503400 is gratefully acknowledged.

\section{References}

[1] Levitt, R.E., "Expert Systems in Construction: State of the Art," in Expert Systems for Civil Engineering: Technology and Applications, American Society of Civil Engineers, 1987.

[2] Hendrickson, C., Zozaya-Gorostiza, C.A., Rehak, D., Baracco-Miller, E.G. and Lim, P.S., "An Expert System for Construction Planning," ASCE Journal of Computing, Vol. 113, No. 5, October 1987.

[3] Zozaya-Gorostiza, C.A. and Hendrickson, C., Knowledge-Based Planning for Construction Projects, Technical Report R-88-170, Department of Civil Engineering, Carnegie-Mellon University, Pittsburgh, PA., April 1988.

[4] Baracco-Miller, E., Planning for Construction, unpublished Master's Thesis, Department of Civil Engr., Carnegie Mellon University, May 1987.

[5] Hendrickson, C., and Au, T., Project Management for Construction: Fundamental Concepts for Owners, Engineers, Architects and Builders, Prentice Hall, Inc., Englewood Cliffs, NJ., 1988.

[6] Hendrickson, C., Martinelli, D. and Rehak, D., "Hierarchical Rule-Based Activity Duration Estimation," ASCE Journal of Construction Engineering and Management, Vol. 113, No. 2, pp. 288-301, June 1987.

[7] Hendrickson, C. and Zozaya-Gorostiza, C.A., A Unified Activity Network Model, Technical Report, Department of Civil Engr., Camegie-Mellon University, Pittsburgh, PA., December 1987.

[8] The Construction Specifications Institute, MASTERFORMAT - Master List of Section Titles and Numbers, Releasing Industry Group, 1983.

[9] Lawler, E., Combinatorial Optimization, Holt-Rinehart and Winston, New York, NY., 1976.

[10] Fenves, S.J., Flemming, U., Hendrickson, C., Maher, M.L. and Schmitt, G. , “An Integrated Software Environment for Building Design and Construction," Proceedings of the Fifth Conference on Computers in Civil Engineering, American Society of Civil Engineers, 1988. 